\title{
Grain-Size Control on the Rare Earth Elements Distribution in the Late Diagenesis of Cretaceous Shales from the Southern Apennines (Italy)
}

\author{
Francesco Cavalcante, ${ }^{1}$ Claudia Belviso, ${ }^{1}$ Giuseppe Piccarreta, ${ }^{2}$ and Saverio Fiore ${ }^{1}$ \\ ${ }^{1}$ Istituto di Metodologie per l'Analisi Ambientale (CNR), Tito Scalo, 85050 Potenza, Italy \\ ${ }^{2}$ Dipartimento Scienze della Terra e Geoambientali, Università di Bari “Aldo Moro", 70125 Bari, Italy \\ Correspondence should be addressed to Francesco Cavalcante; francesco.cavalcante@imaa.cnr.it
}

Received 3 May 2013; Revised 23 October 2013; Accepted 22 November 2013; Published 3 February 2014

Academic Editor: Marc Visseaux

Copyright (c) 2014 Francesco Cavalcante et al. This is an open access article distributed under the Creative Commons Attribution License, which permits unrestricted use, distribution, and reproduction in any medium, provided the original work is properly cited.

Different grain-size fractions of samples collected from shales were investigated to determine the role of late diagenetic grade and mineralogy on the rare earth elements (REE) distribution. The samples were collected from Upper Cretaceous varicoloured shales of the Sicilide Unit near the Corleto Perticara villages, Southern Apennines (Italy). The mineralogical and chemical composition of the sample's five fractions $(>63,32-63,2-32,0.1-2$, and $<0.1 \mu \mathrm{m})$ was studied. The data indicate that certain accessory minerals are more important than clay minerals in controlling the REE distribution. In particular, zircon controls the distribution of earth elements in the $0.1-2 \mu \mathrm{m}$ fraction and is an efficient mechanism in determining the concentration and distribution of REEs in the studied shales.

\section{Introduction}

The rare earth elements (REE) are considered immobile during diagenesis, hydrothermal processes, and metamorphism (e.g., [1-4]). For this reason, the REEs are useful indicators of provenance of dust aerosol (e.g., [5-10]) and fluvial and marine sediments (e.g., [11-13]). However, some studies have provided evidence of the mobilisation and fractionation of REEs during diagenesis and hydrothermal alteration (e.g., $[5,14-19])$ and that these processes depend on the minerals in which the trace elements are hosted. Although it is generally assumed that the rare earth elements are concentrated in the clay-size fraction, a few studies have addressed the role of grain size on REE distribution (e.g., [2, 20-24]), and only some papers have determined the phases in which these elements are mainly hosted $[3,23]$. To our knowledge, no investigations have been carried out on the $<0.1 \mu \mathrm{m}$ grain-size fraction.

Accessory minerals such as monazite, apatite, and zircon, which are common in sedimentary rocks, are known to concentrate and fractionate REEs [11, 25-31]. Other studies, however, have indicated that these minerals cannot control the REE distribution in the sediments $[1,18,32,33]$. González López et al. [34] showed that clay minerals have some influence on REE abundance, whereas other authors suggested that kaolinite is the principal REE carrier in the clay fraction [35]. Additionally, Honty et al. [33] considered authigenic mineral phases concentrated in fine and ultrafine fractions as the most appropriate to address potential REE fractionation induced by either diagenetic or hydrothermal processes. The investigations performed by these authors suggest that accessory minerals had no significant impact on the REE contents in the shales and that the distribution patterns of the various analysed size fractions consist mainly, if not exclusively, of mixed layers of illite-smectite, according to the data in the literature $[1,19]$.

The behaviour of the REEs in clay sediments is not yet completely defined, most likely because such studies were performed on different types of sedimentary rocks with variable amounts of different-sized detrital phases from various geological environments. 
Our study aims to contribute to this discussion by evaluating the effect of distinct grain-size fractions of samples subjected to late diagenesis on the distribution of the REEs. In particular, we focus on the clay fraction $(0.1-2 \mu \mathrm{m}$ and $<0.1 \mu \mathrm{m})$ characterised by the presence of mainly I/S mixed layers. The samples studied were collected from shales characterised by a high amount of ordered illite-smectite mixed layers (I/S; $\mathrm{I}=70-80 \% ; \mathrm{R} 1)$ and showing late diagenesis maturity [36]. Geochemical, mineralogical, and petrographic compositions indicate that a composite crystalline basement, including low- and high-grade metamorphic units, was a major source of these shales, together with some contributions from mafic-ultramafic rocks [37].

\section{Samples and Methods}

Six samples were collected from the Upper Cretaceous varicoloured shales of the Sicilide Unit near the Corleto Perticara villages in the Southern Apennines (Italy) ([38] and references therein) (Figure 1).

Mineralogical and chemical compositions of the $>63$, $32-63,2-32,0.1-2$, and $<0.1 \mu \mathrm{m}$ size fractions were studied. Grain-size separation was performed by wet sieving for the $32-63 \mu \mathrm{m}$ and $>63 \mu \mathrm{m}$ fractions and by gravity settling in distilled water for the clay fraction $(<2 \mu \mathrm{m})$. The splitting of this fraction into two grain-size classes $(<0.1$ and $0.1-2 \mu \mathrm{m})$ was performed by centrifugation, and they were collected by evaporation to avoid any loss of ultrafine particles.

The mineralogy of the whole rock and of the five grain size fractions on random specimens prepared by the side-loading method was determined by X-ray powder diffraction (XRD) using a Siemens D5000 diffractometer $(\mathrm{Cu}-\mathrm{K} \alpha$ radiation; graphite secondary monochromator; sample spinner; step size 0.02 ; speed $0.6^{\circ} / \mathrm{min}$ ). Oriented mounts were prepared by settling a suspension (concentration: $10 \mathrm{mg} / \mathrm{cm}^{2}$ ) on a glass slide after being saturated with $\mathrm{Mg}^{++}$cations using a $1 \mathrm{~N} \mathrm{MgCl}$ solution. Each specimen was analysed in an airdried state, glycolated at $60^{\circ} \mathrm{C}$ for $8 \mathrm{~h}$, and heated at $375^{\circ} \mathrm{C}$ for $1 \mathrm{~h} \mathrm{[39].} \mathrm{The} \mathrm{mixed} \mathrm{layer} \mathrm{I/S} \mathrm{was} \mathrm{identified} \mathrm{following}$ the indications of Moore and Reynolds [39], and a very good agreement exists between our experimental profile and the theoretical profile calculated with Newmod [40]. The comparison showed that there is an excellent agreement between profiles.

Semiquantitative mineralogical analyses of the different fractions, except for the $>63$ and $0.1 \mu \mathrm{m}$ fractions, were performed on random powders and orientated specimens using the WINFIT computer program [41]. The strongest reflection of each mineral was considered. An exception was made for quartz, for which the $4.26 \AA$ line was used instead of the peak at $3.34 \AA$ because of its superimposition with $10 \AA$-minerals and the I-S mixed layer series. The amount of phyllosilicates was estimated by measuring the $4.5 \AA$ peak area. The percentage of phyllosilicates, determined on the oriented specimens, was split according to the following peak areas: $10-15 \AA$ (illite-smectite), $10 \AA$ (illite + micas), and $7 \AA$ (kaolinite + chlorite) minerals. The amount of these latter phases was estimated on the basis of the intensity of

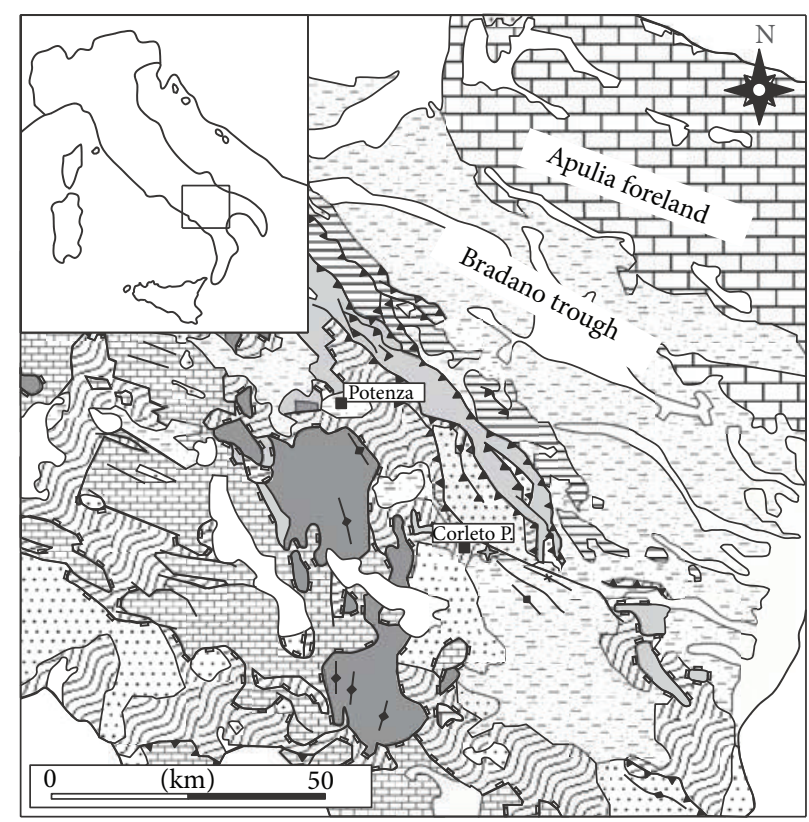

Continental and subordinate coastal deposits (Holocenemiddle Pleistocene p.p.).

Thrust-top

deposits. Terrigenous marine

and paralic deposits filling the Bradano trough and unconformable overlying the Apennine units (middle Pleistocene p.p.-Pliocene).

Miocene thrust-top

siliciclastic deposits

(Castelvetere, Gorgoglione, and Albidona formations).

Internal units: North-

Calabrian and Sicilide units (lower Miocene-Cretaceous).

Alburno-Cervati, Monti della Maddalena derived from the Apennine carbonate platform (lower Miocene-upper Triassic).
Sannio unit (middle

Miocene-lower Cretaceous).

Lagonegro units (lower Cretaceous-middle Triassic).

Tufillo-Serra Palazzo and Daunia units: limestones, calcarenites, and calcirudites followed by siliciclastic turbidites (middle-upper Miocene).

Cretaceous platform carbonates of the Apulia foreland.

- Normal and strike-slip faults

$\checkmark$ Low-angle thrust

4 High-angle thrust

a Anticline axis

* Syncline axis

$\uparrow$ Samples site
FIGURE 1: Geological sketch map of Southern Apennine (modified after [47]) with indication of studied area.

the lines at $3.58 \AA$ and $3.54 \AA$, respectively. A quantitative analysis of the mineralogical phases in the sand fraction was performed only on random powders, whereas the $<0.1$ fraction was studied only on the oriented mounts. The quantitative mineralogical composition of the bulk samples was determined by $\sum$ clay minerals following the method of Laviano [42]. The morphological analyses were performed using scanning electron microscopy (SEM).

The chemical analyses of all samples were performed using ICP-AES (major and minor elements) and ICP-MS (REE, $\mathrm{Y}, \mathrm{Zr}, \mathrm{Nb}, \mathrm{Th}$ ) after fusion of the samples by $\mathrm{LiBO}_{2}$ and $\mathrm{HNO}_{3}$ dissolution. 
TABLE 1: Mineralogical composition of the bulk sample and different fractions.

\begin{tabular}{|c|c|c|c|c|c|c|c|c|c|}
\hline Sample & Qtz & Feld & Cal & Dol & Hem & $\mathrm{I} / \mathrm{S}$ & Ill & Chl & Kao \\
\hline \multicolumn{10}{|c|}{ Bulk sample } \\
\hline $2 \mathrm{~T}$ & 12 & 1 & $\operatorname{tr}$ & 0 & 0 & 50 & 5 & 20 & 12 \\
\hline $4 \mathrm{~T}$ & 11 & 1 & $\operatorname{tr}$ & 1 & 0 & 62 & 2 & 11 & 12 \\
\hline $6 \mathrm{~T}$ & 14 & 7 & 24 & 3 & 0 & 30 & 4 & 14 & 4 \\
\hline $7 \mathrm{~T}$ & 14 & 3 & 2 & 0 & $\operatorname{tr}$ & 58 & 12 & 11 & $\operatorname{tr}$ \\
\hline $8 \mathrm{~T}$ & 18 & 1 & 0 & 0 & 1 & 40 & 6 & 12 & 22 \\
\hline $9 \mathrm{~T}$ & 14 & 1 & 2 & 0 & $\operatorname{tr}$ & 46 & 1 & 15 & 21 \\
\hline \multicolumn{10}{|l|}{ Fractions } \\
\hline \multicolumn{10}{|c|}{$<0.1 \mu \mathrm{m}$} \\
\hline $2 \mathrm{~A}$ & 0 & 0 & 0 & 0 & 0 & 100 & 0 & 0 & $\operatorname{tr}$ \\
\hline $4 \mathrm{~A}$ & 0 & 0 & 0 & 0 & 0 & 100 & 0 & 0 & $\operatorname{tr}$ \\
\hline $6 \mathrm{~A}$ & n.a. & n.a. & n.a. & n.a. & n.a. & n.a. & n.a. & n.a. & n.a. \\
\hline $7 \mathrm{~A}$ & 0 & 0 & 0 & 0 & 0 & 100 & 0 & 0 & 0 \\
\hline $8 \mathrm{~A}$ & 0 & 0 & 0 & 0 & 0 & 100 & 0 & 0 & 0 \\
\hline $9 \mathrm{~A}$ & 0 & 0 & 0 & 0 & 0 & 100 & 0 & 0 & $\operatorname{tr}$ \\
\hline \multicolumn{10}{|c|}{$(0.1-2) \mu \mathrm{m}$} \\
\hline $2 \mathrm{~B}$ & 4 & $\operatorname{tr}$ & 0 & 0 & 0 & 62 & 3 & 14 & 17 \\
\hline $4 \mathrm{~B}$ & 7 & $\operatorname{tr}$ & 1 & 0 & 0 & 74 & 1 & 6 & 11 \\
\hline $6 \mathrm{~B}$ & 6 & $\operatorname{tr}$ & 11 & 0 & 0 & 56 & 3 & 21 & 3 \\
\hline $7 \mathrm{~B}$ & 3 & 1 & 0 & 0 & 0 & 82 & 7 & 6 & 1 \\
\hline $8 \mathrm{~B}$ & 7 & $\operatorname{tr}$ & 0 & 0 & 4 & 58 & 2 & 15 & 14 \\
\hline $9 \mathrm{~B}$ & 3 & $\operatorname{tr}$ & 0 & 0 & 0 & 51 & 5 & 19 & 21 \\
\hline \multicolumn{10}{|c|}{$(2-32) \mu \mathrm{m}$} \\
\hline $2 \mathrm{C}$ & 18 & 2 & 0 & 0 & 0 & 37 & 6 & 28 & 10 \\
\hline $4 \mathrm{C}$ & 18 & 3 & 1 & 0 & $\operatorname{tr}$ & 37 & 2 & 20 & 18 \\
\hline $6 \mathrm{C}$ & 16 & 6 & 25 & 4 & $\operatorname{tr}$ & 21 & 12 & 9 & 6 \\
\hline $7 \mathrm{C}$ & 23 & 5 & 0 & 0 & 1 & 34 & 16 & 20 & 1 \\
\hline $8 \mathrm{C}$ & 31 & 5 & 0 & 0 & 1 & 15 & 6 & 9 & 33 \\
\hline $9 \mathrm{C}$ & 27 & 2 & 0 & 3 & 0 & 22 & 2 & 13 & 31 \\
\hline \multicolumn{10}{|c|}{$(32-63) \mu \mathrm{m}$} \\
\hline $2 \mathrm{D}$ & 20 & 3 & 0 & 0 & 0 & 26 & 7 & 35 & 9 \\
\hline $4 \mathrm{D}$ & n.a. & n.a. & n.a. & n.a. & n.a. & n.a. & n.a. & n.a. & n.a. \\
\hline $6 \mathrm{D}$ & 15 & 10 & 51 & 7 & 0 & $\operatorname{tr}$ & 5 & 5 & 7 \\
\hline $7 \mathrm{D}$ & n.a. & n.a. & n.a. & n.a. & n.a. & n.a. & n.a. & n.a. & n.a. \\
\hline $8 \mathrm{D}$ & 27 & 8 & 0 & 0 & 0 & 18 & 3 & 20 & 24 \\
\hline $9 \mathrm{D}$ & n.a. & n.a. & n.a. & n.a. & n.a. & n.a. & n.a. & n.a. & n.a. \\
\hline \multicolumn{10}{|c|}{$>63 \mu \mathrm{m}$} \\
\hline $2 \mathrm{E}$ & 21 & 2 & 2 & 0 & 0 & 0 & 2 & 60 & 13 \\
\hline $4 \mathrm{E}$ & n.a. & n.a. & n.a. & n.a. & n.a. & n.a. & n.a. & n.a. & n.a. \\
\hline $6 \mathrm{E}$ & 18 & 3 & 64 & 0 & 0 & 0 & 0 & 14 & 0 \\
\hline $7 \mathrm{E}$ & 41 & 5 & 23 & 0 & 0 & 0 & 0 & 30 & 0 \\
\hline $8 \mathrm{E}$ & 24 & 3 & 2 & 0 & 0 & 0 & 9 & 30 & 32 \\
\hline $9 \mathrm{E}$ & 24 & $\operatorname{tr}$ & 43 & 0 & 0 & 0 & 0 & 14 & 19 \\
\hline
\end{tabular}

Qtz: quartz; Feld: K-feldspar + plagioclase; Cal: calcite; Dol: dolomite; Hem: hematite; I/S: illite-smectite mixed layers; Ill: illite and/or muscovite; Chl: chlorite; Kao: kaolinite and dikite; n.a.: not analysed; tr: trace.

\section{Result}

The XRD analysis shows that the bulk samples are mainly composed of clay minerals and quartz (Figure 2); one sample (\#6) is also rich in secondary calcite. Feldspars, dolomite, and hematite are minor constituents. I/S $(\chi=40 \%) R 1$ with $I \%=$ $70-80$ prevails over chlorite $(\chi=15 \%)$, kaolinite $(\chi=14 \%)$, and illite (Ill; $\chi=5 \%$ ) (Table 1 ). I/S is the main constituent of the finest grain-size fraction, and it decreases as grain size increases; it is absent in the coarser fraction. Quartz, 
TABLE 2: Chemical composition (wt\%) of the bulk sample and different fractions.

\begin{tabular}{|c|c|c|c|c|c|c|c|c|c|c|c|}
\hline Sample & $\mathrm{SiO}_{2}$ & $\mathrm{TiO}_{2}$ & $\mathrm{Al}_{2} \mathrm{O}_{3}$ & $\mathrm{Fe}_{2} \mathrm{O}_{3}$ & $\mathrm{MnO}$ & $\mathrm{MgO}$ & $\mathrm{CaO}$ & $\mathrm{Na}_{2} \mathrm{O}$ & $\mathrm{K}_{2} \mathrm{O}$ & $\mathrm{P}_{2} \mathrm{O}_{5}$ & LOI \\
\hline \multicolumn{12}{|c|}{ Bulk sample } \\
\hline $2 \mathrm{~T}$ & 54.38 & 0.86 & 20.26 & 8.01 & 0.03 & 2.89 & 0.51 & 0.86 & 2.48 & 0.30 & 9.17 \\
\hline $4 \mathrm{~T}$ & 54.87 & 1.06 & 19.39 & 7.43 & 0.07 & 1.89 & 1.63 & 0.90 & 2.68 & 0.16 & 9.71 \\
\hline $6 \mathrm{~T}$ & 41.35 & 0.60 & 13.05 & 6.11 & 0.21 & 2.30 & 15.90 & 1.20 & 1.67 & 0.29 & 17.12 \\
\hline $7 \mathrm{~T}$ & 56.14 & 0.78 & 18.79 & 7.38 & 0.03 & 2.63 & 0.94 & 1.06 & 4.27 & 0.14 & 7.61 \\
\hline $8 \mathrm{~T}$ & 58.54 & 0.93 & 19.90 & 7.53 & 0.02 & 2.11 & 0.24 & 1.06 & 2.03 & 0.17 & 7.28 \\
\hline $9 \mathrm{~T}$ & 52.54 & 1.20 & 21.23 & 7.78 & 0.07 & 1.59 & 2.47 & 1.24 & 1.51 & 0.16 & 9.99 \\
\hline \multicolumn{12}{|l|}{ Fractions } \\
\hline \multicolumn{12}{|c|}{$<0.1 \mu \mathrm{m}$} \\
\hline $2 \mathrm{~A}$ & 46.65 & 0.04 & 23.46 & 4.75 & 0.00 & 2.69 & 0.46 & 4.26 & 3.74 & 0.62 & 13.06 \\
\hline $4 \mathrm{~A}$ & 41.08 & 0.07 & 20.63 & 4.01 & 0.00 & 1.95 & 1.82 & 7.60 & 4.55 & 0.05 & 18.00 \\
\hline $6 \mathrm{~A}$ & n.a. & n.a. & n.a. & n.a. & n.a. & n.a. & n.a. & n.a. & n.a. & n.a. & n.a. \\
\hline $7 \mathrm{~A}$ & 41.51 & 0.05 & 19.42 & 5.02 & 0.00 & 2.16 & 0.57 & 7.24 & 5.20 & 0.05 & 17.70 \\
\hline $8 \mathrm{~A}$ & 45.92 & 0.04 & 25.65 & 4.96 & 0.00 & 2.62 & 0.32 & 2.84 & 4.05 & 0.35 & 13.05 \\
\hline $9 \mathrm{~A}$ & 37.72 & 0.04 & 22.65 & 1.62 & 0.00 & 1.24 & 1.47 & 11.37 & 3.01 & 0.05 & 19.68 \\
\hline \multicolumn{12}{|c|}{$(0.1-2) \mu \mathrm{m}$} \\
\hline $2 \mathrm{~B}$ & 48.69 & 1.10 & 24.87 & 7.06 & 0.02 & 2.90 & 0.66 & 0.64 & 3.37 & 0.37 & 10.04 \\
\hline $4 \mathrm{~B}$ & 52.59 & 1.34 & 22.83 & 5.67 & 0.03 & 1.74 & 0.95 & 0.43 & 4.08 & 0.16 & 9.94 \\
\hline $6 \mathrm{~B}$ & 43.50 & 0.95 & 20.77 & 7.87 & 0.08 & 2.85 & 6.98 & 0.44 & 3.39 & 0.42 & 12.52 \\
\hline $7 \mathrm{~B}$ & 50.71 & 0.84 & 23.18 & 7.21 & 0.00 & 2.86 & 0.47 & 0.41 & 5.72 & 0.16 & 8.18 \\
\hline $8 B$ & 47.65 & 1.27 & 24.57 & 10.47 & 0.02 & 2.91 & 0.29 & 0.65 & 3.18 & 0.19 & 8.56 \\
\hline $9 \mathrm{~B}$ & 47.40 & 1.61 & 26.87 & 7.39 & 0.02 & 1.64 & 0.58 & 0.94 & 2.45 & 0.17 & 10.66 \\
\hline \multicolumn{12}{|c|}{$(2-32) \mu \mathrm{m}$} \\
\hline $2 \mathrm{C}$ & 58.85 & 0.77 & 17.92 & 8.88 & 0.03 & 2.89 & 0.39 & 0.66 & 1.96 & 0.20 & 7.21 \\
\hline $4 \mathrm{C}$ & 56.75 & 0.96 & 17.68 & 9.45 & 0.09 & 2.04 & 1.63 & 0.82 & 1.85 & 0.13 & 8.43 \\
\hline $6 C$ & 46.92 & 0.60 & 12.63 & 6.48 & 0.18 & 2.44 & 12.90 & 1.42 & 1.34 & 0.27 & 14.63 \\
\hline $7 \mathrm{C}$ & 63.53 & 0.82 & 15.13 & 7.94 & 0.02 & 2.43 & 0.35 & 1.15 & 2.71 & 0.11 & 5.61 \\
\hline $8 \mathrm{C}$ & 65.66 & 0.82 & 17.10 & 6.15 & 0.00 & 1.59 & 0.16 & 1.10 & 1.25 & 0.10 & 5.88 \\
\hline $9 \mathrm{C}$ & 58.62 & 1.10 & 18.64 & 8.54 & 0.07 & 1.58 & 1.11 & 0.87 & 0.90 & 0.14 & 8.26 \\
\hline \multicolumn{12}{|c|}{$(32-63) \mu \mathrm{m}$} \\
\hline $2 \mathrm{D}$ & 50.62 & 0.64 & 19.64 & 13.08 & 0.05 & 3.84 & 0.52 & 0.50 & 1.87 & 0.24 & 8.72 \\
\hline $4 \mathrm{D}$ & n.a. & n.a. & n.a. & n.a. & n.a. & n.a. & n.a. & n.a. & n.a. & n.a. & n.a. \\
\hline $6 \mathrm{D}$ & 33.04 & 0.17 & 5.92 & 4.22 & 0.24 & 1.43 & 28.64 & 0.84 & 0.44 & 0.11 & 24.80 \\
\hline $7 \mathrm{D}$ & n.a. & n.a. & n.a. & n.a. & n.a. & n.a. & n.a. & n.a. & n.a. & n.a. & n.a. \\
\hline $8 \mathrm{D}$ & 62.23 & 0.65 & 16.32 & 9.06 & 0.03 & 2.32 & 0.43 & 1.04 & 1.39 & 0.14 & 6.21 \\
\hline $9 \mathrm{D}$ & n.a. & n.a. & n.a. & n.a. & n.a. & n.a. & n.a. & n.a. & n.a. & n.a. & n.a. \\
\hline \multicolumn{12}{|c|}{$>63 \mu \mathrm{m}$} \\
\hline $2 \mathrm{E}$ & 46.02 & 0.53 & 19.54 & 16.38 & 0.23 & 4.57 & 1.00 & 0.37 & 1.60 & 0.21 & 9.33 \\
\hline $4 \mathrm{E}$ & n.a. & n.a. & n.a. & n.a. & n.a. & n.a. & n.a. & n.a. & n.a. & n.a. & n.a. \\
\hline $6 \mathrm{E}$ & 20.06 & 0.19 & 4.88 & 3.43 & 0.36 & 1.16 & 37.46 & 0.39 & 0.55 & 0.14 & 31.16 \\
\hline $7 \mathrm{E}$ & 50.38 & 0.43 & 10.64 & 8.57 & 0.37 & 2.77 & 11.43 & 0.71 & 1.30 & 0.12 & 13.04 \\
\hline $8 \mathrm{E}$ & 53.52 & 0.54 & 17.45 & 13.47 & 0.17 & 3.24 & 1.60 & 0.56 & 1.23 & 0.15 & 7.87 \\
\hline $9 \mathrm{E}$ & 28.52 & 0.42 & 9.43 & 10.91 & 0.42 & 1.04 & 23.85 & 0.33 & 0.54 & 0.11 & 22.97 \\
\hline
\end{tabular}

calcite, and chlorite are concentrated in the coarser fraction (>63 $\mu \mathrm{m})$, whereas kaolinite, illite, feldspars, and dolomite are more abundant in the silt fractions $(2-32 \mu \mathrm{m}$ and $32-63 \mu \mathrm{m})$ (Table 1).

The major element abundance is given in Table 2. The chemical compositions of the bulk samples are close to those of the PAAS (Post-Archean Average Australian Shale; McLennan, 1989) (Figure 3), except for $\mathrm{CaO}$ and $\mathrm{MnO}$, which are scattered. In fact, although the general chemical composition of the bulk samples is quite uniform, the amount of $\mathrm{CaO}$ is variable $(\min .=0.42 \%, \max .=15.90 \%)$ according to the presence of secondary calcite identified in some samples. The 
TABLE 3: The trace elements abundance (ppm) of the bulk sample and different fractions.

\begin{tabular}{|c|c|c|c|c|c|c|c|c|c|c|c|c|c|c|c|}
\hline Sample & $\mathrm{Ba}$ & $\mathrm{Be}$ & $\mathrm{Co}$ & $\mathrm{Cr}$ & $\mathrm{Cu}$ & $\mathrm{Ga}$ & $\mathrm{Nb}$ & $\mathrm{Ni}$ & $\mathrm{Rb}$ & Sc & $\mathrm{Sr}$ & Th & $\mathrm{Y}$ & $\mathrm{Zn}$ & $\mathrm{Zr}$ \\
\hline \multicolumn{16}{|c|}{ Bulk sample } \\
\hline $2 \mathrm{~T}$ & 303.0 & 3.4 & 20.7 & 187.0 & 72.5 & 30.1 & 27.5 & 69.3 & 124.0 & 12.1 & 297.0 & 13.7 & 26.7 & 145.0 & 197.0 \\
\hline $4 \mathrm{~T}$ & 189.0 & 4.3 & 19.3 & 122.0 & 33.8 & 31.7 & 28.7 & 51.3 & 147.0 & 12.6 & 181.0 & 13.8 & 34.0 & 106.0 & 198.0 \\
\hline $6 \mathrm{~T}$ & 143.0 & 2.3 & 11.5 & 133.0 & 45.7 & 19.1 & 11.2 & 38.6 & 79.5 & 12.6 & 353.0 & 8.4 & 20.6 & 89.0 & 86.6 \\
\hline $7 \mathrm{~T}$ & 272.0 & 3.8 & 17.8 & 148.0 & 81.4 & 26.7 & 13.5 & 65.1 & 184.0 & 13.3 & 190.0 & 12.2 & 21.3 & 93.9 & 128.0 \\
\hline $8 \mathrm{~T}$ & 153.0 & 3.7 & 17.7 & 145.0 & 56.2 & 26.8 & 17.0 & 55.2 & 96.4 & 12.3 & 126.0 & 11.2 & 20.8 & 92.8 & 140.0 \\
\hline $9 \mathrm{~T}$ & 131.0 & 3.2 & 21.6 & 128.0 & 38.5 & 29.8 & 33.8 & 63.1 & 75.2 & 13.1 & 249.0 & 14.4 & 29.7 & 113.0 & 237.0 \\
\hline \multicolumn{16}{|l|}{ Fractions } \\
\hline \multicolumn{16}{|c|}{$<0.5 \mu \mathrm{m}$} \\
\hline $2 \mathrm{~A}$ & 353.0 & 4.2 & 15.9 & 207.0 & 103.0 & 39.3 & 1.9 & 65.9 & 184.0 & 13.8 & 382.0 & 5.4 & 18.2 & 139.0 & 161.0 \\
\hline $4 \mathrm{~A}$ & 237.0 & 4.0 & 10.8 & 124.0 & 47.6 & 42.6 & 2.0 & 34.4 & 223.0 & 11.8 & 507.0 & 5.1 & 15.3 & 83.2 & 101.0 \\
\hline $6 \mathrm{~A}$ & n.a. & n.a. & n.a. & n.a. & n.a. & n.a. & n.a. & n.a. & n.a. & n.a. & n.a. & n.a. & n.a. & n.a. & n.a. \\
\hline $7 \mathrm{~A}$ & 205.0 & 4.1 & 8.4 & 151.0 & 77.1 & 27.7 & 0.8 & 36.7 & 196.0 & 12.1 & 222.0 & 2.0 & 3.9 & 82.3 & 30.8 \\
\hline $8 \mathrm{~A}$ & 150.0 & 3.9 & 12.1 & 226.0 & 114.0 & 46.9 & 0.8 & 62.0 & 180.0 & 19.1 & 193.0 & 2.6 & 6.1 & 110.0 & 31.4 \\
\hline $9 \mathrm{~A}$ & 176.0 & 4.3 & 8.0 & 132.0 & 43.0 & 47.5 & 1.1 & 24.6 & 124.0 & 12.2 & 726.0 & 5.0 & 17.4 & 78.7 & 148.0 \\
\hline \multicolumn{16}{|c|}{$(0.5-2) \mu \mathrm{m}$} \\
\hline $2 \mathrm{~B}$ & 356.0 & 3.7 & 17.7 & 210.0 & 71.9 & 37.8 & 36.3 & 65.9 & 171.0 & 15.1 & 385.0 & 19.6 & 36.9 & 139.0 & 234.0 \\
\hline $4 \mathrm{~B}$ & 278.0 & 5.1 & 18.2 & 135.0 & 32.9 & 41.9 & 35.1 & 42.6 & 219.0 & 13.6 & 235.0 & 17.5 & 42.1 & 81.7 & 241.0 \\
\hline $6 \mathrm{~B}$ & 265.0 & 3.1 & 16.9 & 219.0 & 64.3 & 34.9 & 18.1 & 54.2 & 159.0 & 13.1 & 266.0 & 13.1 & 28.5 & 141.0 & 132.0 \\
\hline $7 \mathrm{~B}$ & 351.0 & 3.3 & 15.0 & 178.0 & 49.8 & 34.0 & 14.8 & 56.6 & 251.0 & 15.6 & 215.0 & 13.5 & 24.2 & 96.7 & 141.0 \\
\hline $8 \mathrm{~B}$ & 229.0 & 3.2 & 23.0 & 210.0 & 70.6 & 38.2 & 24.4 & 67.7 & 157.0 & 17.7 & 195.0 & 14.8 & 27.9 & 128.0 & 180.0 \\
\hline $9 B$ & 200.0 & 3.9 & 23.0 & 161.0 & 38.3 & 43.7 & 44.9 & 63.3 & 123.0 & 14.1 & 377.0 & 20.1 & 42.2 & 131.0 & 334.0 \\
\hline \multicolumn{16}{|c|}{$(2-32) \mu \mathrm{m}$} \\
\hline $2 \mathrm{C}$ & 232.0 & 2.2 & 20.4 & 156.0 & 68.9 & 23.1 & 23.5 & 68.0 & 93.1 & 10.0 & 218.0 & 10.3 & 20.8 & 162.0 & 162.0 \\
\hline $4 \mathrm{C}$ & 150.0 & 2.8 & 23.4 & 113.0 & 35.1 & 25.0 & 25.3 & 63.9 & 93.4 & 11.8 & 118.0 & 10.8 & 28.4 & 136.0 & 163.0 \\
\hline $6 \mathrm{C}$ & 131.0 & 2.0 & 12.5 & 120.0 & 47.8 & 16.9 & 11.2 & 39.6 & 59.3 & 11.8 & 225.0 & 8.4 & 18.0 & 95.8 & 89.5 \\
\hline $7 \mathrm{C}$ & 218.0 & 2.4 & 21.2 & 120.0 & 70.7 & 20.4 & 14.0 & 76.1 & 109.0 & 9.3 & 106.0 & 10.2 & 19.9 & 104.0 & 129.0 \\
\hline $8 \mathrm{C}$ & 122.0 & 2.0 & 15.8 & 109.0 & 36.7 & 19.5 & 15.4 & 41.9 & 58.8 & 8.1 & 93.7 & 8.8 & 19.6 & 86.0 & 136.0 \\
\hline $9 \mathrm{C}$ & 102.0 & 1.8 & 20.8 & 111.0 & 36.1 & 22.0 & 31.4 & 67.4 & 45.2 & 11.3 & 136.0 & 11.1 & 24.9 & 118.0 & 195.0 \\
\hline \multicolumn{16}{|c|}{$(32-63) \mu \mathrm{m}$} \\
\hline $2 \mathrm{D}$ & 470.0 & 2.4 & 31.5 & 194.0 & 179.0 & 26.4 & 19.3 & 96.7 & 84.0 & 10.8 & 294.0 & 9.6 & 20.7 & 219.0 & 139.0 \\
\hline $4 \mathrm{D}$ & n.a. & n.a. & n.a. & n.a. & n.a. & n.a. & n.a. & n.a. & n.a. & n.a. & n.a. & n.a. & n.a. & n.a. & n.a. \\
\hline $6 \mathrm{D}$ & 56.5 & 0.6 & 8.6 & 54.3 & 58.7 & 6.5 & 3.3 & 23.5 & 21.5 & 12.8 & 421.0 & 2.8 & 15.0 & 54.0 & 25.7 \\
\hline $7 \mathrm{D}$ & n.a. & n.a. & n.a. & n.a. & n.a. & n.a. & n.a. & n.a. & n.a. & n.a. & n.a. & n.a. & n.a. & n.a. & n.a. \\
\hline $8 \mathrm{D}$ & 127.0 & 2.0 & 23.3 & 117.0 & 133.0 & 22.0 & 11.5 & 55.1 & 63.4 & 9.6 & 138.0 & 7.8 & 16.8 & 120.0 & 96.3 \\
\hline $9 \mathrm{D}$ & n.a. & n.a. & n.a. & n.a. & n.a. & n.a. & n.a. & n.a. & n.a. & n.a. & n.a. & n.a. & n.a. & n.a. & n.a. \\
\hline \multicolumn{16}{|c|}{$>63 \mu \mathrm{m}$} \\
\hline $2 \mathrm{E}$ & 327.0 & 2.2 & 32.2 & 164.0 & 178.0 & 26.8 & 16.2 & 105.0 & 72.8 & 11.8 & 249.0 & 7.9 & 16.7 & 278.0 & 119.0 \\
\hline $4 \mathrm{E}$ & n.a. & n.a. & n.a. & n.a. & n.a. & n.a. & n.a. & n.a. & n.a. & n.a. & n.a. & n.a. & n.a. & n.a. & n.a. \\
\hline $6 \mathrm{E}$ & 54.3 & 1.1 & 5.0 & 48.0 & 26.9 & 7.5 & 4.0 & 23.4 & 26.4 & 11.8 & 940.0 & 3.3 & 20.0 & 39.9 & 32.4 \\
\hline $7 \mathrm{E}$ & 281.0 & 1.3 & 22.6 & 62.1 & 272.0 & 14.4 & 8.1 & 73.1 & 56.7 & 14.6 & 324.0 & 5.5 & 26.0 & 134.0 & 102.0 \\
\hline $8 \mathrm{E}$ & 117.0 & 1.6 & 35.2 & 101.0 & 346.0 & 26.1 & 10.7 & 69.7 & 61.8 & 12.1 & 133.0 & 6.3 & 14.6 & 181.0 & 100.0 \\
\hline $9 \mathrm{E}$ & 72.4 & 1.1 & 53.6 & 48.7 & 65.8 & 13.7 & 12.5 & 87.8 & 29.2 & 17.8 & 422.0 & 4.6 & 30.6 & 173.0 & 94.9 \\
\hline
\end{tabular}

slightly higher $\mathrm{Fe}$ content is most likely due to the presence of $\mathrm{Fe}$-chlorite and the lower amount of $\mathrm{K}_{2} \mathrm{O}$ to a scarcity of feldspars, illite, and/or micas. The chemical compositions of the different grain-size fractions indicate that $\mathrm{SiO}_{2}$ is concentrated in the $2-32 \mu \mathrm{m}$ fraction; $\mathrm{Al}_{2} \mathrm{O}_{3}$ is concentrated in the fine fraction $(<2 \mu \mathrm{m})$, in accordance with the higher amount of clay minerals; and $\mathrm{TiO}_{2}$ and $\mathrm{P}_{2} \mathrm{O}_{5}$ are concentrated in the $0.1-2 \mu \mathrm{m}$ fraction. $\mathrm{Fe}_{2} \mathrm{O}_{3}, \mathrm{MnO}, \mathrm{MgO}, \mathrm{CaO}$, and LOI show higher values in the $>63 \mu \mathrm{m}$ fraction than in the finer fractions, corresponding to the higher amount of Fe-chlorite 
TABLE 4: The REE abundance (ppm) of the bulk sample and different fractions.

\begin{tabular}{|c|c|c|c|c|c|c|c|c|c|c|c|c|c|c|}
\hline Sample & $\mathrm{La}$ & $\mathrm{Ce}$ & $\operatorname{Pr}$ & $\mathrm{Nd}$ & $\mathrm{Sm}$ & $\mathrm{Eu}$ & $\mathrm{Gd}$ & $\mathrm{Tb}$ & Dy & Ho & $\mathrm{Er}$ & $\mathrm{Tm}$ & $\mathrm{Yb}$ & $\mathrm{Lu}$ \\
\hline \multicolumn{15}{|c|}{ Bulk sample } \\
\hline $2 \mathrm{~T}$ & 50.2 & 101.8 & 10.6 & 40.9 & 7.7 & 1.7 & 6.4 & 0.9 & 5.0 & 1.0 & 2.7 & 0.4 & 2.6 & 0.4 \\
\hline $4 \mathrm{~T}$ & 47.9 & 98.5 & 11.2 & 43.7 & 8.8 & 2.0 & 7.6 & 1.2 & 6.4 & 1.4 & 3.3 & 0.5 & 3.2 & 0.4 \\
\hline $6 \mathrm{~T}$ & 32.3 & 55.7 & 6.9 & 25.9 & 4.7 & 1.0 & 3.8 & 0.6 & 3.3 & 0.8 & 2.0 & 0.3 & 1.8 & 0.3 \\
\hline $7 \mathrm{~T}$ & 33.3 & 71.0 & 8.1 & 30.4 & 5.4 & 1.1 & 4.4 & 0.7 & 3.7 & 0.8 & 2.2 & 0.4 & 2.4 & 0.4 \\
\hline $8 \mathrm{~T}$ & 31.1 & 72.9 & 7.4 & 28.4 & 5.3 & 1.2 & 4.2 & 0.7 & 3.7 & 0.9 & 2.1 & 0.3 & 2.3 & 0.3 \\
\hline $9 \mathrm{~T}$ & 51.0 & 107.5 & 12.3 & 47.5 & 8.9 & 1.8 & 7.1 & 1.1 & 6.2 & 1.3 & 3.2 & 0.5 & 3.4 & 0.5 \\
\hline \multicolumn{15}{|l|}{ Fractions } \\
\hline \multicolumn{15}{|c|}{$<0.1 \mu \mathrm{m}$} \\
\hline $2 \mathrm{~A}$ & 18.8 & 35.0 & 3.5 & 12.2 & 2.3 & 0.6 & 2.2 & 0.4 & 2.6 & 0.7 & 1.9 & 0.3 & 2.3 & 0.4 \\
\hline $4 \mathrm{~A}$ & 14.6 & 27.0 & 2.8 & 9.6 & 1.7 & 0.4 & 1.6 & 0.3 & 2.3 & 0.6 & 1.5 & 0.3 & 1.5 & 0.2 \\
\hline $6 \mathrm{~A}$ & n.a. & n.a. & n.a. & n.a. & n.a. & n.a. & n.a. & n.a. & n.a. & n.a. & n.a. & n.a. & n.a. & n.a. \\
\hline $7 \mathrm{~A}$ & 4.4 & 7.3 & 0.7 & 2.3 & 0.4 & 0.1 & 0.5 & 0.1 & 0.6 & 0.2 & 0.4 & 0.1 & 0.5 & 0.1 \\
\hline $8 \mathrm{~A}$ & 7.1 & 14.0 & 1.3 & 4.7 & 1.0 & 0.3 & 1.0 & 0.1 & 0.9 & 0.2 & 0.6 & 0.1 & 0.6 & 0.1 \\
\hline $9 \mathrm{~A}$ & 7.8 & 14.8 & 1.4 & 4.6 & 0.9 & 0.2 & 1.1 & 0.3 & 2.4 & 0.7 & 2.0 & 0.3 & 2.0 & 0.3 \\
\hline \multicolumn{15}{|c|}{$(0.1-2) \mu \mathrm{m}$} \\
\hline $2 \mathrm{~B}$ & 75.4 & 148.1 & 15.8 & 60.8 & 11.0 & 2.4 & 9.0 & 1.3 & 7.4 & 1.5 & 3.9 & 0.6 & 3.6 & 0.6 \\
\hline $4 \mathrm{~B}$ & 73.2 & 143.9 & 15.4 & 61.9 & 12.1 & 2.5 & 9.8 & 1.4 & 8.4 & 1.8 & 4.4 & 0.6 & 3.9 & 0.6 \\
\hline $6 \mathrm{~B}$ & 64.5 & 107.0 & 12.7 & 43.9 & 7.3 & 1.5 & 6.0 & 0.9 & 5.2 & 1.0 & 2.7 & 0.4 & 2.8 & 0.4 \\
\hline $7 \mathrm{~B}$ & 45.4 & 90.6 & 10.0 & 37.5 & 6.6 & 1.3 & 5.0 & 0.7 & 4.3 & 0.9 & 2.5 & 0.4 & 2.9 & 0.4 \\
\hline $8 \mathrm{~B}$ & 55.2 & 122.5 & 12.1 & 46.2 & 7.7 & 1.5 & 5.8 & 0.9 & 5.3 & 1.1 & 2.8 & 0.4 & 3.3 & 0.5 \\
\hline $9 \mathrm{~B}$ & 83.8 & 167.0 & 18.1 & 70.2 & 12.5 & 2.2 & 8.5 & 1.3 & 8.2 & 1.8 & 4.9 & 0.7 & 5.0 & 0.7 \\
\hline \multicolumn{15}{|c|}{$(2-32) \mu \mathrm{m}$} \\
\hline $2 \mathrm{C}$ & 38.3 & 77.4 & 8.7 & 32.4 & 6.0 & 1.3 & 4.7 & 0.7 & 4.1 & 0.8 & 2.2 & 0.3 & 2.3 & 0.3 \\
\hline $4 \mathrm{C}$ & 38.2 & 76.7 & 8.7 & 32.8 & 7.4 & 1.7 & 6.5 & 1.0 & 5.3 & 1.1 & 2.6 & 0.4 & 2.5 & 0.4 \\
\hline $6 \mathrm{C}$ & 27.5 & 47.9 & 6.0 & 22.4 & 4.6 & 0.9 & 3.7 & 0.5 & 3.1 & 0.7 & 1.6 & 0.2 & 1.6 & 0.2 \\
\hline $7 \mathrm{C}$ & 27.9 & 56.8 & 6.3 & 24.1 & 4.9 & 1.0 & 4.0 & 0.6 & 3.4 & 0.8 & 2.0 & 0.3 & 2.1 & 0.3 \\
\hline $8 \mathrm{C}$ & 23.0 & 53.0 & 5.3 & 19.4 & 4.6 & 1.0 & 3.6 & 0.6 & 3.5 & 0.8 & 1.9 & 0.3 & 1.9 & 0.3 \\
\hline $9 \mathrm{C}$ & 39.3 & 79.1 & 9.2 & 36.4 & 7.5 & 1.6 & 6.3 & 1.0 & 5.1 & 1.0 & 2.6 & 0.4 & 2.3 & 0.3 \\
\hline \multicolumn{15}{|c|}{$(32-63) \mu \mathrm{m}$} \\
\hline $2 \mathrm{D}$ & 36.5 & 71.0 & 8.1 & 30.8 & 5.4 & 1.3 & 4.4 & 0.7 & 3.8 & 0.8 & 2.0 & 0.3 & 1.9 & 0.3 \\
\hline $4 \mathrm{D}$ & n.a. & n.a. & n.a. & n.a. & n.a. & n.a. & n.a. & n.a. & n.a. & n.a. & n.a. & n.a. & n.a. & n.a. \\
\hline $6 \mathrm{D}$ & 11.6 & 20.6 & 2.9 & 10.8 & 2.3 & 0.6 & 2.1 & 0.3 & 2.1 & 0.5 & 1.2 & 0.2 & 1.2 & 0.2 \\
\hline $7 \mathrm{D}$ & n.a. & n.a. & n.a. & n.a. & n.a. & n.a. & n.a. & n.a. & n.a. & n.a. & n.a. & n.a. & n.a. & n.a. \\
\hline $8 \mathrm{D}$ & 20.2 & 44.0 & 4.7 & 18.3 & 4.0 & 0.9 & 3.5 & 0.6 & 3.1 & 0.7 & 1.6 & 0.2 & 1.5 & 0.2 \\
\hline $9 \mathrm{D}$ & n.a. & n.a. & n.a. & n.a. & n.a. & n.a. & n.a. & n.a. & n.a. & n.a. & n.a. & n.a. & n.a. & n.a. \\
\hline \multicolumn{15}{|c|}{$>63 \mu \mathrm{m}$} \\
\hline $2 \mathrm{E}$ & 30.1 & 58.6 & 6.6 & 25.0 & 4.5 & 1.0 & 3.6 & 0.5 & 3.1 & 0.7 & 1.6 & 0.3 & 1.6 & 0.2 \\
\hline $4 \mathrm{E}$ & n.a. & n.a. & n.a. & n.a. & n.a. & n.a. & n.a. & n.a. & n.a. & n.a. & n.a. & n.a. & n.a. & n.a. \\
\hline $6 \mathrm{E}$ & 12.9 & 23.9 & 3.2 & 12.9 & 2.8 & 0.7 & 2.8 & 0.4 & 2.5 & 0.6 & 1.5 & 0.2 & 1.3 & 0.2 \\
\hline $7 \mathrm{E}$ & 12.7 & 29.3 & 3.5 & 15.0 & 4.7 & 1.2 & 4.5 & 0.8 & 4.4 & 0.9 & 2.2 & 0.3 & 2.0 & 0.3 \\
\hline $8 \mathrm{E}$ & 16.1 & 37.9 & 3.7 & 13.9 & 2.9 & 0.6 & 2.4 & 0.4 & 2.4 & 0.5 & 1.3 & 0.2 & 1.4 & 0.2 \\
\hline $9 \mathrm{E}$ & 18.1 & 39.1 & 4.4 & 17.5 & 5.5 & 1.7 & 6.8 & 1.2 & 5.8 & 1.1 & 2.3 & 0.3 & 1.7 & 0.2 \\
\hline
\end{tabular}

and calcite. Instead, $\mathrm{Na}_{2} \mathrm{O}$ and $\mathrm{K}_{2} \mathrm{O}$ are concentrated in the fine fraction $(<0.1 \mu \mathrm{m})$, in accordance with the presence of natroiarosite and a higher amount of $\mathrm{I} / \mathrm{S}$, respectively.

The trace element distributions are given in Tables 3 and 4. The $32-63 \mu \mathrm{m}$ fraction shows enrichment in $\mathrm{Cu}$ compared to the other fractions, whereas the $<0.1$ and $0.1-2 \mu \mathrm{m}$ fractions are more enriched in $\mathrm{Ba}, \mathrm{Be}, \mathrm{Cr}, \mathrm{Ga}, \mathrm{Rb}$, and $\mathrm{Sr}$ than the coarser fractions, most likely because of the adsorption of these elements by clay minerals. The amounts of Co, Ni, and $\mathrm{Zn}$ are comparable to each other, with a downward trend in the fine fraction $(0.1 \mu \mathrm{m})$. Sc shows enrichment in the fineultrafine fraction, but $\mathrm{Nb}, \mathrm{Th}, \mathrm{Y}$, and $\mathrm{Zr}$ are characterised by 
TABLE 5: Correlation matrix for same elements. LREE $=(\mathrm{La}-\mathrm{Nd}) ; \mathrm{MREE}=(\mathrm{Sm}-\mathrm{Tb}) ; \mathrm{HREE}=(\mathrm{Dy}-\mathrm{Lu})$. Bold numbers indicate $P>0.001$; italic numbers indicate $0.01<P<0.001$.

(a) Bulk sample

\begin{tabular}{|c|c|c|c|c|c|c|c|c|}
\hline & LREE & MREE & HREE & $\mathrm{Nb}$ & Th & Y & $\mathrm{Zr}$ & $\mathrm{P}_{2} \mathrm{O}_{5}$ \\
\hline LREE & 1.0000 & & & & & & & \\
\hline MREE & 0.9675 & 1.0000 & & & & & & \\
\hline HREE & 0.9319 & 0.9848 & 1.0000 & & & & & \\
\hline $\mathrm{Nb}$ & 0.9841 & 0.9624 & 0.9424 & 1.0000 & & & & \\
\hline Th & 0.9177 & 0.8762 & 0.8677 & 0.8792 & 1.0000 & & & \\
\hline $\mathrm{Y}$ & 0.8764 & 0.9681 & 0.9617 & 0.8740 & 0.7702 & 1.0000 & & \\
\hline $\mathrm{Zr}$ & 0.9780 & 0.9376 & 0.9270 & 0.9851 & 0.9370 & 0.8263 & 1.0000 & \\
\hline $\mathrm{P}_{2} \mathrm{O}_{5}$ & -0.1173 & -0.2067 & -0.3540 & -0.1509 & -0.3922 & -0.2184 & -0.2546 & 1.0000 \\
\hline
\end{tabular}

(b) Fraction $>63 \mu \mathrm{m}$

\begin{tabular}{|c|c|c|c|c|c|c|c|c|}
\hline & LREE & MREE & HREE & $\mathrm{Nb}$ & Th & $\mathrm{Y}$ & $\mathrm{Zr}$ & $\mathrm{P}_{2} \mathrm{O}_{5}$ \\
\hline LREE & 1.0000 & & & & & & & \\
\hline MREE & 0.1925 & 1.0000 & & & & & & \\
\hline HREE & 0.0126 & 0.9684 & 1.0000 & & & & & \\
\hline $\mathrm{Nb}$ & 0.9244 & 0.3873 & 0.2330 & 1.0000 & & & & \\
\hline Th & 0.8284 & -0.0548 & -0.1273 & 0.8314 & 1.0000 & & & \\
\hline $\mathrm{Y}$ & -0.2724 & 0.8817 & 0.9307 & -0.0929 & -0.4784 & 1.0000 & & \\
\hline $\mathrm{Zr}$ & 0.6876 & 0.3704 & 0.3398 & 0.8569 & 0.8661 & -0.0261 & 1.0000 & \\
\hline $\mathrm{P}_{2} \mathrm{O}_{5}$ & 0.7896 & -0.4188 & -0.5561 & 0.5486 & 0.7451 & -0.7425 & 0.3369 & 1.0000 \\
\hline
\end{tabular}

(c) Fraction (32-63) $\mu \mathrm{m}$

\begin{tabular}{|c|c|c|c|c|c|c|c|c|}
\hline & LREE & MREE & HREE & $\mathrm{Nb}$ & Th & $\mathrm{Y}$ & $\mathrm{Zr}$ & $\mathrm{P}_{2} \mathrm{O}_{5}$ \\
\hline LREE & 1.0000 & & & & & & & \\
\hline MREE & 0.9839 & 1.0000 & & & & & & \\
\hline HREE & 0.9895 & 0.9994 & 1.0000 & & & & & \\
\hline $\mathrm{Nb}$ & 0.9930 & 0.9981 & 0.9996 & 1.0000 & & & & \\
\hline Th & 0.9336 & 0.9826 & 0.9756 & 0.9694 & 1.0000 & & & \\
\hline $\mathrm{Y}$ & 0.9943 & 0.9592 & 0.9684 & 0.9747 & 0.8901 & 1.0000 & & \\
\hline $\mathrm{Zr}$ & 0.9704 & 0.9979 & 0.9951 & 0.9921 & 0.9925 & 0.9391 & 1.0000 & \\
\hline $\mathrm{P}_{2} \mathrm{O}_{5}$ & 0.9803 & 0.9292 & 0.9415 & 0.9501 & 0.8445 & 0.9958 & 0.9036 & 1.0000 \\
\hline
\end{tabular}

(d) Fraction (0.1-2) $\mu \mathrm{m}$

\begin{tabular}{lccccccc}
\hline & LREE & MREE & HREE & $\mathrm{Nb}$ & $\mathrm{Th}$ & $\mathrm{Y}$ & $\mathrm{Zr}$ \\
\hline LREE & 1.0000 & & & & & & \\
$\mathrm{MREE}$ & 0.9337 & 1.0000 & & & & & \\
$\mathrm{HREE}$ & 0.9620 & 0.9666 & 1.0000 & & & & \\
$\mathrm{Nb}$ & $\mathbf{0 . 9 8 5 8}$ & 0.9272 & 0.9672 & 1.0000 & & & \\
$\mathrm{Th}$ & 0.9375 & 0.9037 & 0.9086 & 0.9667 & 1.0000 & & \\
$\mathrm{Y}$ & 0.9501 & $\mathbf{0 . 9 8 4 8}$ & $\mathbf{0 . 9 8 9 7}$ & 0.9380 & 0.8780 & 1.0000 & 1.0000 \\
$\mathrm{Zr}$ & 0.9364 & 0.8526 & 0.9447 & 0.9715 & 0.9265 & 0.8899 & -0.3380 \\
$\mathrm{P}_{2} \mathrm{O}_{5}$ & -0.0469 & -0.1090 & -0.2443 & -0.1808 & -0.1185 & -0.1601 & 1.0000 \\
\hline
\end{tabular}

(e) Finest fraction $(<0.1) \mu \mathrm{m}$

\begin{tabular}{lccccccc}
\hline & LREE & MREE & HREE & $\mathrm{Nb}$ & $\mathrm{Th}$ & $\mathrm{Y}$ & $\mathrm{Zr}$ \\
\hline LREE & 1.0000 & & & & & & \\
$\mathrm{MREE}$ & $\mathbf{0 . 9 9 3 1}$ & 1.0000 & & & & \\
$\mathrm{HREE}$ & 0.7402 & 0.7521 & 1.0000 & & & \\
$\mathrm{Nb}$ & 0.9308 & 0.8860 & 0.7233 & 1.0000 & & & \\
$\mathrm{Th}$ & 0.8038 & 0.8041 & 0.9788 & 0.8193 & 1.0000 & & \\
\hline
\end{tabular}


(e) Continued.

\begin{tabular}{lcccccccc}
\hline & LREE & MREE & HREE & $\mathrm{Nb}$ & $\mathrm{Th}$ & $\mathrm{Y}$ & $\mathrm{Zr}$ & $\mathrm{P}_{2} \mathrm{O}_{5}$ \\
\hline $\mathrm{Y}$ & 0.7535 & 0.7628 & 0.9972 & 0.7483 & 0.9905 & 1.0000 & \\
$\mathrm{Zr}$ & 0.6996 & 0.7172 & 0.9867 & 0.6567 & 0.9349 & 0.9717 & 1.0000 \\
$\mathrm{P}_{2} \mathrm{O}_{5}$ & 0.6115 & 0.6755 & 0.2555 & 0.2913 & 0.2263 & 0.2350 & 0.2990 & 1.0000 \\
\hline
\end{tabular}

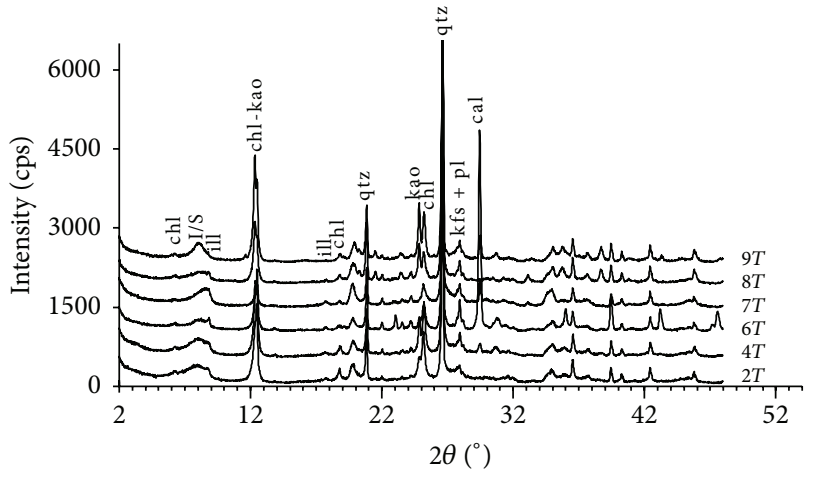

FIGURE 2: XRD patterns of bulk sample. Chl = chlorite; Kao = kaolinite; Ill = illite; I/S = illite-smectite mixed layers; $\mathrm{Qtz}=$ quartz; $\mathrm{Kfs}+\mathrm{Pl}=\mathrm{K}$-feldspar + plagioclase $; \mathrm{Cal}=$ calcite .

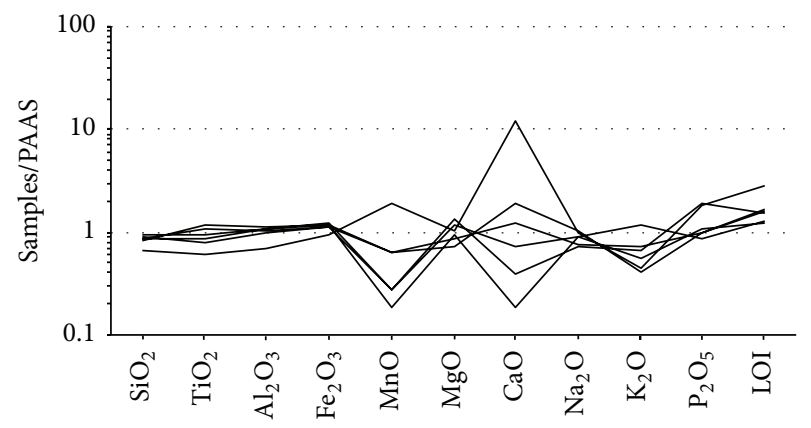

FIGURE 3: PAAS-normalized major elements patterns.

a higher value of the clay fraction $(0.1-2 \mu \mathrm{m})$ compared with the other grain-size fractions, particularly with the $<0.1 \mu \mathrm{m}$ fraction. The REE distribution in the whole rock is similar to that of the PAAS, except for a slight enrichment in MREEs $(\mathrm{Sm}-\mathrm{Tb})$ and a depletion in HREEs (Dy-Lu) (Figure 4). The elemental concentration in the 5 grain-size fractions, normalised to the whole rock, shows that the concentration decreases rapidly from the $0.1-2 \mu \mathrm{m}$ fraction (up to $1.5 \mathrm{X}$ ) to the $2-32 \mu \mathrm{m}(0.8 \mathrm{X}), 32-63 \mu \mathrm{m}$, and $>63 \mu \mathrm{m}$ fractions (approximately $0.5 \mathrm{X}$ ); the lowest concentration was observed in the finest fraction $(<0.1 \mu \mathrm{m} ; 0.2 \mathrm{X})$.

\section{Discussion}

The samples studied show a highly homogeneous chemical composition comparable with the PAAS. The mineralogical composition is typical of clays that underwent a late diagenetic grade. The very low content of REEs measured in the finest grain-size fraction $\left(\sum \mathrm{REE}=17-83 \mathrm{ppm}\right)$ clearly indicates that this fraction is not the carrier of REEs and that only very small amounts of these elements, if any, are hosted by the I/S mixed layer. This hypothesis is not in accordance with some literature data indicating REEs are essentially concentrated in kaolinite minerals ([20] and references therein). A strong linear correlation (probability $P$ varying from 0.01 to 0.001 ) exists between the HREEs (Dy to $\mathrm{Lu}$ ) and $\mathrm{Zr}$, Th, and $\mathrm{Y}$ (Table 5), suggesting that the presence and distribution of HREEs in the fine fraction of the studied shales are controlled by zircon (e.g. [26, 29]). The similarity between the pattern of this mineral and that of the finest fraction confirms this hypothesis. Thus, an enrichment of REE content does not correspond to the high specific surface of the finest fraction, suggesting that adsorption is not an efficient mechanism for determining the concentration and distribution of these elements in the studied shales.

The highest REE content in the $0.1-2 \mu \mathrm{m}$ grain-size fraction does not appear to be related to the clay minerals, but there is a strong correlation between REEs and $\mathrm{Zr}$ (not observed in the finest grain-size fraction) and between $\mathrm{Y}$, $\mathrm{Zr}$, REEs, and Nb. This result suggests that accessory phases may be more important than clay minerals in controlling the distribution of the REEs in shales, which is particularly evident from the comparison of the REE-Nb-Th-Zr-Y distribution in the distinct grain-size fractions of the studied shales (Figure 4). The higher the concentration of the REE contents, the higher the concentration of the other trace elements. In addition, strong similarities exist between the Y, HREE, and MREE profiles and between the Th and LREE profiles.

The data are also confirmed by the abundance of isolated zircon $(1-2 \mu \mathrm{m})$ sometimes associated with kaolinite crystals (Figure 5). In our opinion, the presence of kaolinite with zircon could be explained taking into account that this clay mineral is an alteration product of zircon carrying phyllosilicates.

Some studies have suggested an influence of REE-rich phosphate on the REE distribution in fine-grained sediments $[43,44]$. Rasmussen [45], for instance, documented the presence of REE-phosphate in marine sandstone and suggested a diagenetic origin. Di Leo and Fiore [46], using selective chemical dissolution techniques, deduced the presence of phosphate containing REEs in shales. Although the studied shales underwent late diagenetic processes, phosphates did not play a significant role in the REE fine fraction distribution. Thus, it appears that the elucidation of the presence/absence of REE-rich phosphate minerals in shales requires further investigation. 


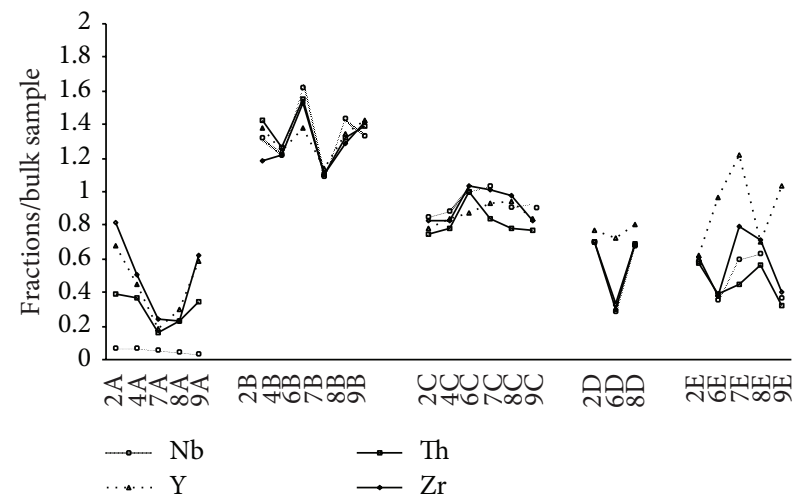

(a)

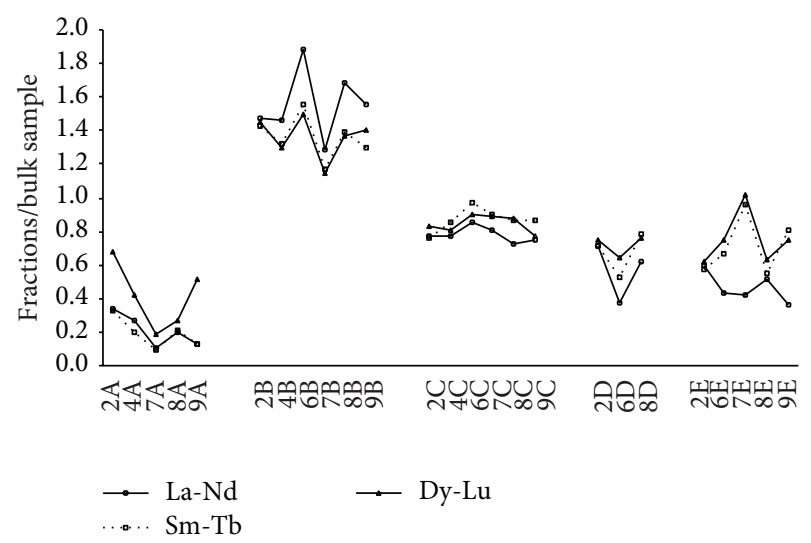

(b)

FIGURE 4: Bulk sample-normalized REE patterns of fractions.

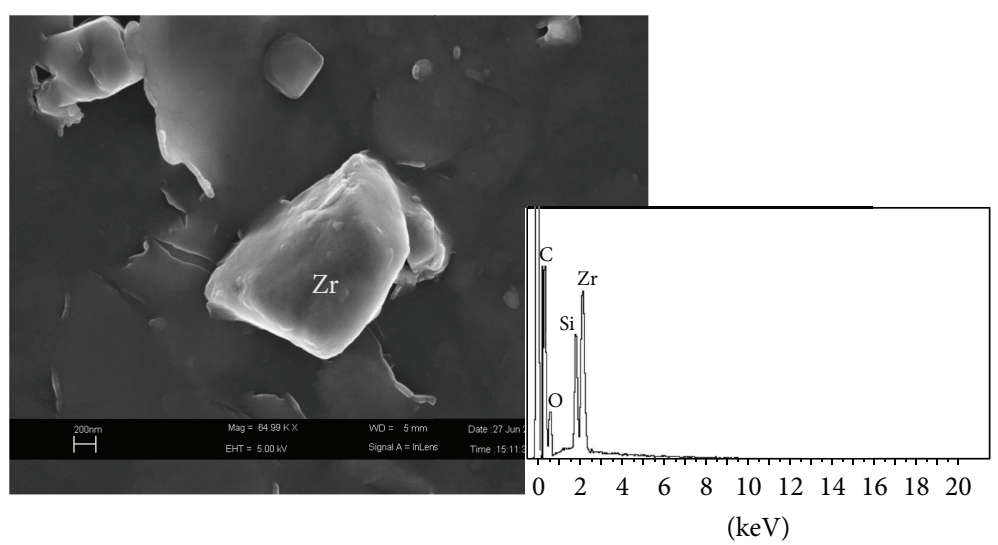

(a)

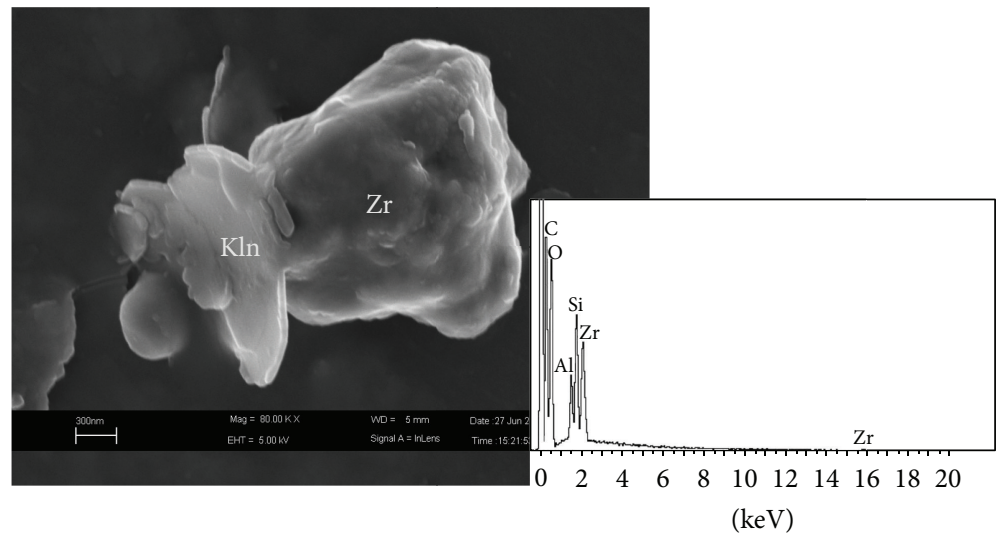

(b)

FIGURE 5: SEM images of (a) single particle of zircon ( $\mathrm{Zr}$ ) and (b) zircon ( $\mathrm{Zr}$ ) and kaolinite (kao) crystals.

\section{Concluding Remarks}

Mineralogical and chemical data of grain-size fractions of 6 samples collected from shales affected by late diagenesis maturity indicate that distribution of REEs in the fine fraction is mainly controlled by zircon. Phosphates did not play an important role in the fine fraction distribution of the REE.
This result is not in accordance with some data available in the literature that indicate the fundamental role assumed by illite-smectite mixed layers or phosphates.

\section{Conflict of Interests}

The authors declare that there is no conflict of interests. 


\section{References}

[1] K. C. Condie, "Another look at rare earth elements in shales," Geochimica et Cosmochimica Acta, vol. 55, no. 9, pp. 2527-2531, 1991.

[2] R. L. Cullers, S. Chaudhuri, B. Arnold, M. Lee, and C. W. Wolf Jr., "Rare earth distributions in clay minerals and in the claysized fraction of the Lower Permian Havensville and Eskridge shales of Kansas and Oklahoma," Geochimica et Cosmochimica Acta, vol. 39, no. 12, pp. 1691-1703, 1975.

[3] R. Cullers, S. Chaudhuri, N. Kilbane, and R. Koch, "Rare-earths in size fractions and sedimentary rocks of PennsylvanianPermian age from the mid-continent of the U.S.A.," Geochimica et Cosmochimica Acta, vol. 43, no. 8, pp. 1285-1301, 1979.

[4] S. R. Taylor and S. M. McLennan, The Continental Crust: Its Composition and Evolution, Blackwell, Oxford, UK, 1985.

[5] D. N. Awwiller and L. E. Mack, "Diagenetic modification of SmNd model ages in Tertiary sandstones and shales, Texas Gulf Coast," Geology, vol. 19, no. 4, pp. 311-314, 1991.

[6] X. P. Yang, Y. S. Liu, C. Z. Li, Y. L. Song, H. P. Zhu, and X. D. Jin, "Rare earth elements of aeolian deposits in Northern China and their implications for determining the provenance of dust storms in Beijing," Geomorphology, vol. 87, no. 4, pp. 365-377, 2007.

[7] C. Zdanowicz, G. Hall, J. Vaive et al., "Asian dustfall in the St. Elias Mountains, Yukon, Canada," Geochimica et Cosmochimica Acta, vol. 70, no. 14, pp. 3493-3507, 2006.

[8] Q. G. Zhang, S. C. Kang, S. Kaspari et al., "Rare earth elements in an ice core from Mt. Everest: seasonal variations and potential sources," Atmospheric Research, vol. 94, no. 2, pp. 300-312, 2009.

[9] C. Li, S. Kang, Q. Zhang, and C. M. Sharma, "Effectiveness of rare earth elements constrain on different materials: a case study in central Asia," Environmental Earth Sciences, vol. 67, no. 5, pp. 1415-1421, 2012.

[10] J. L. Feng, Z. G. Hu, J. T. Ju, and L. P. Zhu, "Variations in trace element (including rare earth element) concentrations with grain sizes in loess and their implications for tracing the provenance of eolian deposits," Quaternary International, vol. 236, no. 1-2, pp. 116-126, 2011.

[11] S. Y. Yang, H. S. Jung, M. S. Choi, and C. X. Li, "The rare earth element compositions of the Changjiang (Yangtze) and Huanghe (Yellow) river sediments," Earth and Planetary Science Letters, vol. 201, no. 2, pp. 407-419, 2002.

[12] D. Z. Piper, R. B. Perkins, and H. D. Rowe, "Rare-earth elements in the permian phosphoria formation: paleo proxies of ocean geochemistry," Deep-Sea Research II, vol. 54, no. 11-13, pp. 13961413, 2007.

[13] P. Censi, A. Incarbona, E. Oliveri, S. Bonomo, and G. Tranchida, "Yttrium and REE signature recognized in Central Mediterranean Sea (ODP site 963) during the MIS 6-MIS 5 transition," Palaeogeography, Palaeoclimatology, Palaeoecology, vol. 292, no. 1-2, pp. 201-210, 2010.

[14] B. Bock, S. M. McLennan, and G. N. Hanson, "Rare earth element redistribution and its effects on the neodymium isotope system in the austin Glen Member of the Normanskill Formation, New York, USA," Geochimica et Cosmochimica Acta, vol. 58, no. 23, pp. 5245-5253, 1994.

[15] P. Fulignati, A. Gioncada, and A. Sbrana, "Rare-earth element (REE) behaviour in the alteration facies of the active magmatichydrothermal system of Vulcano (Aeolian Islands, Italy)," Journal of Volcanology and Geothermal Research, vol. 88, no. 4, pp. 325-342, 1999.
[16] A. E. Milodowski and J. A. Zalasiewicz, "Redistribution of rare earth elements during diagenesis of turbidite/hemipelagite mudrock sequences of Landovery age from central Walles," in Developments in Sedimentary Provenance Studies, A. C. Morton, S. P. Todd, and P. D. W. Haughton, Eds., vol. 57, pp. 101-124, Geological Society of London, Special Publication, London, UK, 1991

[17] M. Ohr, A. N. Halliday, and D. R. Peacor, "Mobility and fractionation of rare earth elements in argillaceous sediments: implications for dating diagenesis and low-grade metamorphism," Geochimica et Cosmochimica Acta, vol. 58, no. 1, pp. 289-312, 1994.

[18] I. T. Uysal and S. D. Golding, "Rare earth element fractionation in authigenic illite-smectite from Late Permian clastic rocks, Bowen Basin, Australia: implications for physico-chemical environments of fluids during illitization," Chemical Geology, vol. 193, no. 3-4, pp. 167-179, 2003.

[19] H. Zwingmann, N. Clauer, and R. Gaupp, "Structure-related geochemical (REE) and isotopic (K-Ar, Rb-Sr, $\delta$ 18O) characteristics of clay minerals from Rotliegend sandstone reservoirs (Permian, northern Germany)," Geochimica et Cosmochimica Acta, vol. 63, no. 18, pp. 2805-2823, 1999.

[20] R. Marques, M. Isabel Dias, M. Isabel Prudêncio, and F. Rocha, "Upper cretaceous clayey levels from western Portugal (Aveiro and Taveiro regions): clay mineral and trace-element distribution," Clays and Clay Minerals, vol. 59, no. 3, pp. 315327, 2011.

[21] R. L. Cullers, T. Barrett, R. Carlson, and B. Robinson, "Rareearth element and mineralogic changes in Holocene soil and stream sediment: a case study in the Wet Mountains, Colorado, U.S.A.," Chemical Geology, vol. 63, no. 3-4, pp. 275-297, 1987.

[22] G. Mongelli, R. L. Cullers, and S. Muelheisen, "Geochemistry of late cretaceous-oligocenic shales from the varicolori formation, southern Apennines, Italy: implications for mineralogical, grain-size control and provenance," European Journal of Mineralogy, vol. 8, no. 4, pp. 733-754, 1996.

[23] G. W. A. Nyakairu and C. Koeberl, "Variation of mineral, chemical, and rare earth element composition in size fractions of clay-rich sediments from the Kajjansi and Ntawo clay deposits, central Uganda," Chemie der Erde, vol. 62, no. 1, pp. 73-86, 2002.

[24] E. Roaldset, "Rare earth elements in different size fractions of a marine quick clay from Ullensaker, and a till from Upper Numedal, Norway," Clay Minerals, vol. 14, no. 3, pp. 229-240, 1979.

[25] B. Bauluz, M. J. Mayayo, C. Fernandez-Nieto, and J. M. G. Lopez, "Geochemistry of Precambrian and Paleozoic siliciclastic rocks from the Iberian Range (NE Spain): implications for source-area weathering, sorting, provenance, and tectonic setting," Chemical Geology, vol. 168, no. 1-2, pp. 135-150, 2000.

[26] A. Caggianelli, S. Fiore, G. Mongelli, and A. Salvemini, "REE distribution in the clay fraction of pelites from the southern Apennines, Italy," Chemical Geology, vol. 99, no. 4, pp. 253-263, 1992.

[27] S. Fiore and G. Mongelli, "Hypothesis on the genesis of clay minerals in the fine fraction of "Argille varicolori" from Andretta (southern Apennines)," Mineralogica et Petrographica Acta, vol. 34, pp. 183-190, 1991.

[28] S. M. McLennan, "Rare earth elements in sedimentary rocks: influence of provenance and sedimentary processes," in Geochemistry and Mineralogy of Rare Earth Elements. Review of 
Mineralogy, B. R. Lipin and G. A. McKay, Eds., vol. 21, pp. 169200, 1989.

[29] X. P. Yan, R. Kerrich, and M. J. Hendry, "Trace element geochemistry of a thick till and clay-rich aquitard sequence, Saskatchewan, Canada," Chemical Geology, vol. 164, no. 1-2, pp. 93-120, 2000.

[30] H. S. Jung, D. I. Lim, S. Y. Yang, and H. S. Yoo, "Constraints of REE distribution patterns in core sediments and their provenance, Northeen East China Sea," Economic and Environmental Geology, vol. 39, pp. 39-51, 2006.

[31] H. S. Jung, D. Lim, J. Y. Choi, H. S. Yoo, K. C. Rho, and H. B. Lee, "Rare earth element compositions of core sediments from the shelf of the South Sea, Korea: their controls and origins," Continental Shelf Research, vol. 48, pp. 75-86, 2012.

[32] G. Q. Cai, F. Guo, X. T. Liu, S. Sui, C. W. Li, and L. Zhao, "Geochemistry of Neogene sedimentary rocks from the Jiyang basin, North China Block: the roles of grain size and clay minerals," Geochemical Journal, vol. 42, no. 5, pp. 381-402, 2008.

[33] M. Honty, N. Clauer, and V. Šucha, "Rare-earth elemental systematics of mixed-layered illite-smectite from sedimentary and hydrothermal environments of the Western Carpathians (Slovakia)," Chemical Geology, vol. 249, no. 1-2, pp. 167-190, 2008.

[34] J. M. González López, B. Bauluz, C. Fernández-Nieto, and A. Y. Oliete, "Factors controlling the trace-element distribution in fine-grained rocks: the Albian kaolinite-rich deposits of the Oliete Basin (NE Spain)," Chemical Geology, vol. 214, no. 1-2, pp. 1-19, 2005.

[35] M. I. Prudencio, M. O. Figueiredo, and J. M. P. Cabral, "Rare earth distribution and its correlation with clay mineralogy in the clay-sized fraction of Cretaceous and Pliocene sediments (central Portugal)," Clay Minerals, vol. 24, no. 1, pp. 67-74, 1989.

[36] F. Cavalcante, S. Fiore, A. Lettino, G. Piccarreta, and F. Tateo, "Illite-smectite mixed layers in sicilide shales and piggy-back deposits of the Gorgoglione Formation (Southern Apennines): geological inferences," Bollettino della Societa Geologica Italiana, vol. 126, no. 2, pp. 241-254, 2007.

[37] F. Cavalcante, S. Fiore, G. Piccarreta, and F. Tateo, "Geochemical and mineralogical approaches to assessing provenance and deposition of shales: a case study," Clay Minerals, vol. 38, no. 3, pp. 383-397, 2003.

[38] F. Lentini, S. Carbone, A. Di Stefano, and P. Guarnieri, "Stratigraphical and structural constraints in the Lucanian Apennines (Southern Italy): tools for reconstructing the geological evolution," Journal of Geodynamics, vol. 34, no. 1, pp. 141-158, 2002.

[39] D. M. Moore and R. C. Reynolds, X-Ray Diffraction and the Identification and Analysis of Clay Minerals, Oxford University Press, Oxford, UK, 2nd edition, 1997.

[40] R. C. Reynolds, "Description of Programm Newmod for the calculation of the one-dimensional X-ray diffraction patterns of mixed-layered clays," 1985.

[41] S. Krumm, "WINFIT 1.2: version of November 1996 (The Erlangen geological and mineralogical software collection) of 'WINFIT 1.0: a public domain program for interactive profileanalysis under WINDOWS', in Proceedings of the 13th Conference on Clay Mineralogy and Petrology in Praha 1994, vol. 38, pp. 253-261, Acta Universitatis Carolinae Geologica, Praha, Czech Republic, 1996.

[42] R. Laviano, "Analisi mineralogica quantitativa di argille mediante diffrattometria di raggi $\mathrm{X}$. In: procedure di analisi di materiali argillosi," in Atti del Workshop Centro Ricerche Energia Ambiente, pp. 215-234, ENEA, S. Teresa, NM, USA, 1987.
[43] J. F. Banfield and R. A. Eggleton, "Apatite replacement and rare earth mobilization, fractionation, and fixation during weathering," Clays \& Clay Minerals, vol. 37, no. 2, pp. 113-127, 1989.

[44] N. K. Foley and R. A. Ayuso, "Rare earth element mobility in high-alumina altered metavolcanic deposits South Carolina, USA," Journal of Geochemical Exploration, vol. 133, pp. 50-67, 2013.

[45] B. Rasmussen, "Early-diagenetic REE-phosphate minerals (florencite, gorceixite, crandallite, and xenotime) in marine sandstones: a major sink for oceanic phosphorus," American Journal of Science, vol. 296, no. 6, pp. 601-632, 1996.

[46] P. Di Leo and S. Fiore, "REE distribution in shales as inferred by chemical selective dissolution," in Proceedings of the 11th International Clay Conference, vol. A-21, Ottawa, Canada, 1997.

[47] E. Patacca and P. Scandone, "Geology of the Southern Apennines," Bollettino della Società Geologica Italiana, vol. 7, pp. 75119, 2007. 

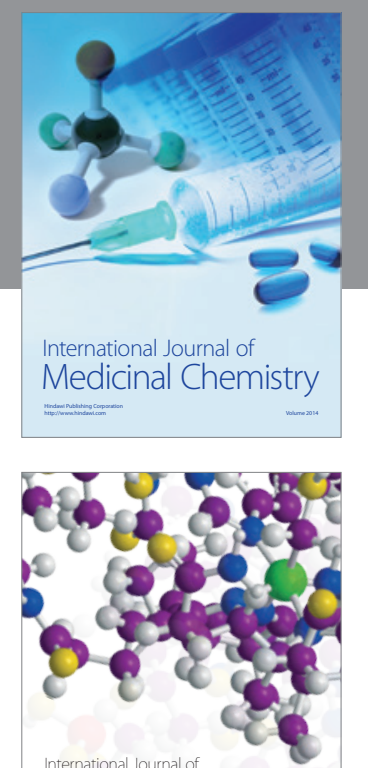

\section{Carbohydrate} Chemistry

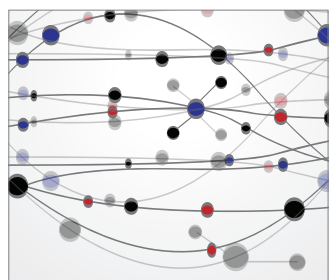

The Scientific World Journal
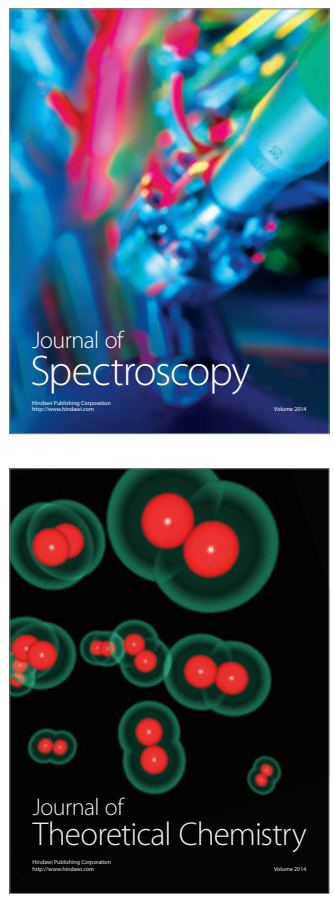
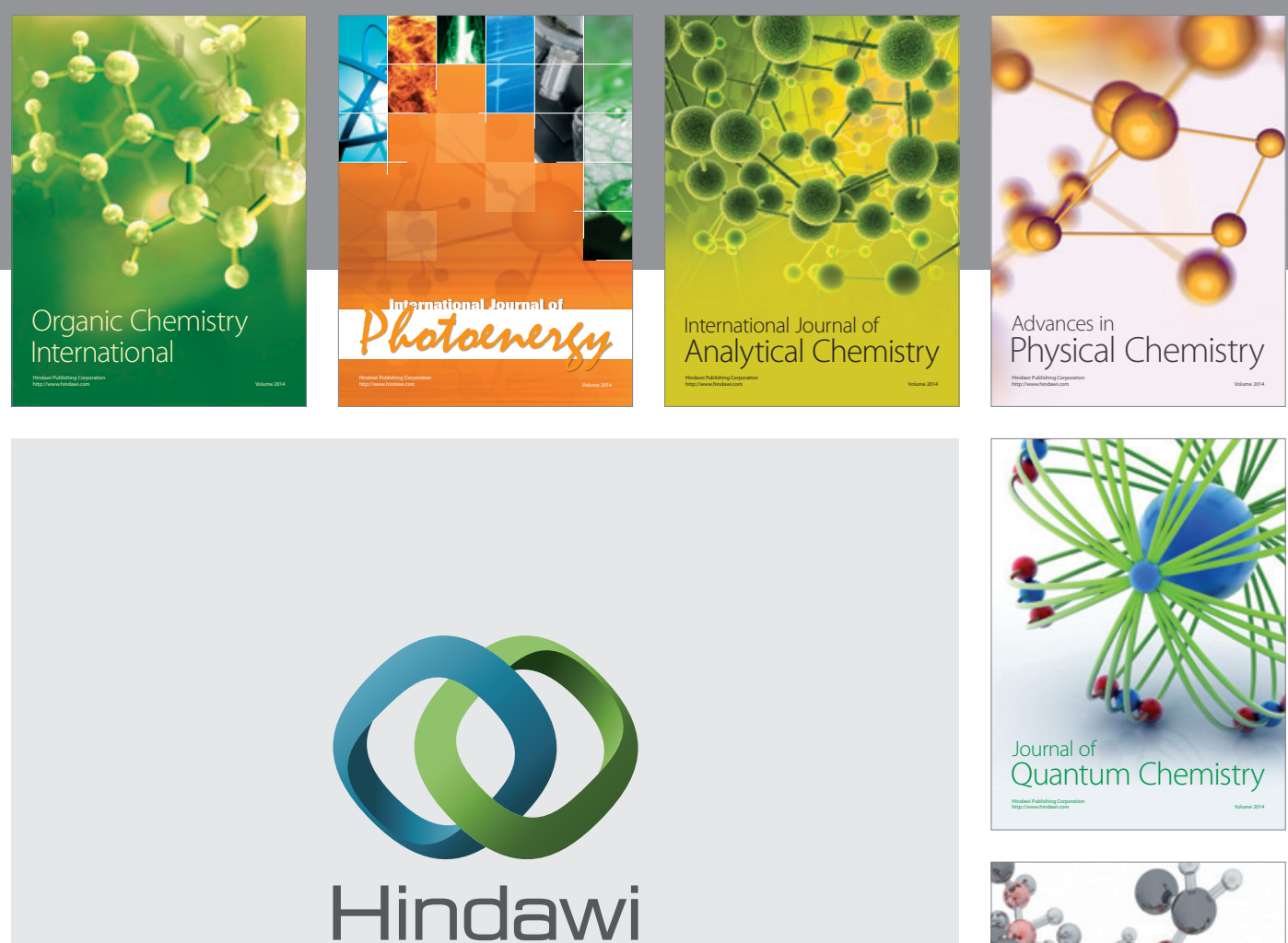

Submit your manuscripts at

http://www.hindawi.com

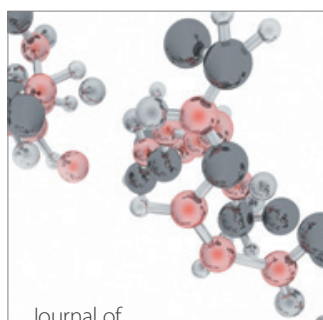

Analytical Methods

in Chemistry

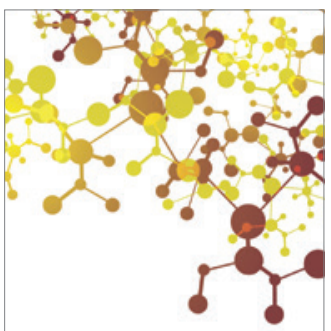

Journal of

Applied Chemistry

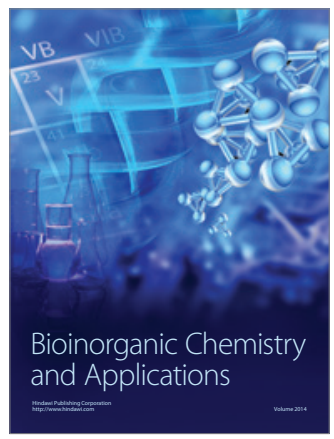

Inorganic Chemistry
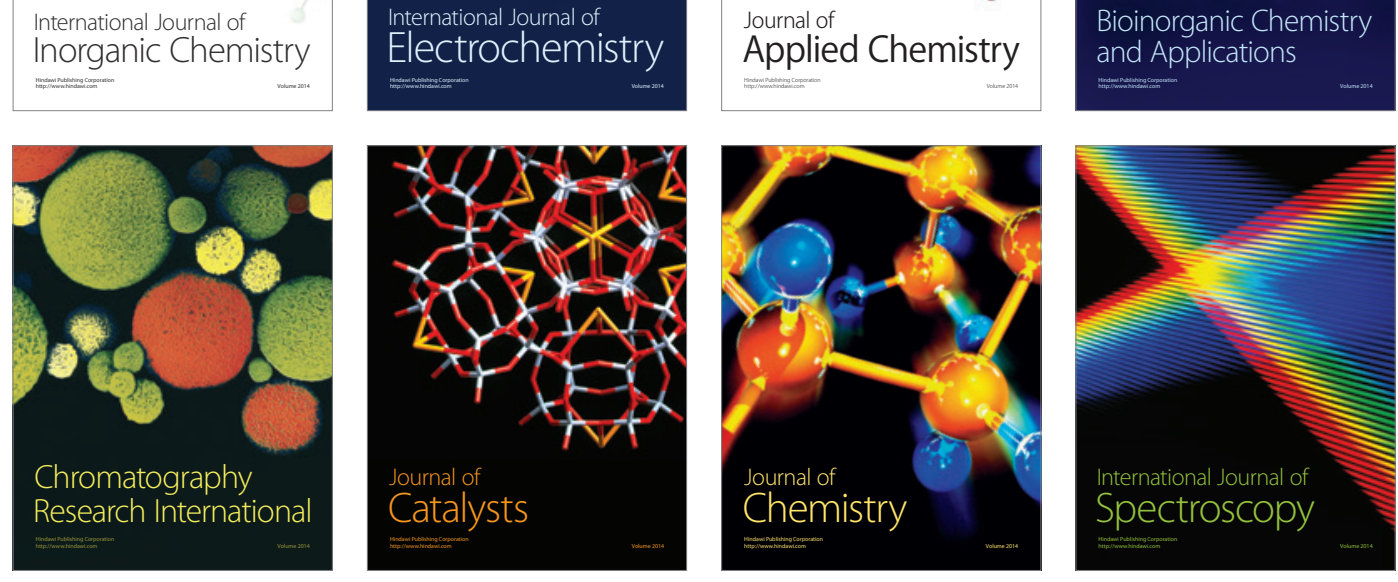